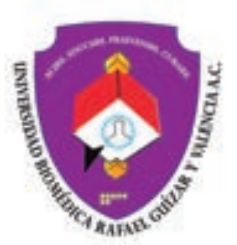

Vol. 8, Núm. 1

Enero-Junio 2021. pp. 13-18

doi: 10.35366/101200

Artículo original

\title{
Vacunación contra SARS-CoV-2: una estrategia global para contender contra la pandemia
}

\section{Vaccination against SARS-CoV-2: a global strategy to cope with the pandemic}

\author{
Jorge Escobedo-de la Peña*
}

\section{RESUMEN}

Objetivo: presentar la información sobre la vacunación contra COVID-19. Material y métodos: se revisaron los datos publicados sobre estudios poblacionales que evalúan la efectividad de la inmunización contra esta enfermedad en diferentes grupos de personas. Resultados: la certeza de la vacuna frente a la COVID-19 varía en las diversas muestras, en gran medida dependiendo de la plataforma usada en el desarrollo de las diferentes marcas. No obstante, en la actualidad se cuenta con una amplia gama de opciones de vacuna con adecuada eficacia, seguridad, que despiertan una buena respuesta inmunológica en la población inyectada, con capacidad efectiva comprobada. Conclusiones: la inoculación universal, aunada a la inmunidad adquirida por el padecimiento y las medidas no farmacológicas para la prevención del contagio, como el uso de cubreboca y el distanciamiento social, contribuirán a disminuir la carga que ha representado esta pandemia en la salud global.

Palabras clave: Vacunación, COVID-19, eficacia, efectividad.

\section{ABSTRACT}

Objective: To present data regarding vaccination against COVID-19. Material and methods: Published information was reviewed regarding population studies assessing the effectiveness of COVID-19 vaccination in different populations. Results: Effectiveness of COVID-19 vaccine varies in diverse populations, mainly due to the platforms employed while developing the vaccines. Nevertheless, currently there is a broad spectrum of vaccine options, with adequate efficacy, safety, immunogenicity, and good effectiveness. Conclusions: Universal vaccination, together with the acquired immunity due to the disease, and nonpharmacological measures to prevent infection, like mask usage and social distancing, will help diminish the burden that this disease has posed to global health.

Keywords: Vaccination, COVID-19, efficacy, effectiveness.

\section{INTRODUCCIÓN}

La pandemia por COVID-19 ha afectado sensiblemente la salud a nivel global, tanto por su elevada contagiosidad, como por su alta letalidad. Si bien hay grupos poblaciona- les claramente definidos con mayor riesgo de morir, por ejemplo las personas adultas longevas, los enfermos de diabetes mellitus o de hipertensión arterial, o los individuos obesos, lo cierto es que aquellos aún sin estas condiciones, están en riesgo de fallecer al contraer la infección. ${ }^{1}$

* Unidad de Investigación en Epidemiología Clínica. Hospital Regional Núm. 1 Carlos MacGregor Sánchez Navarro, IMSS.

Correspondencia:

Jorge Escobedo-de la Peña

Gabriel Mancera Núm. 222, Col. Del Valle, 03100, Alcaldía Benito Juárez, CDMX. Tel. y Fax: 5255 5087-5871.

E-mail: jorge.escobedo@imss.gob.mx

Recibido: 03-08-2021. Aceptado: 13-08-2021.

Citar como: Escobedo-de la Peña J. Vacunación contra SARS-CoV-2: una estrategia global para contender contra la pandemia. Plast Restaur Neurol. 2021;8 (1): 13-18. https://dx.doi.org/10.35366/101200 
Desde inicios de la epidemia, se han planteado acciones poblacionales a fin de reducir las posibilidades de contagio, como son el uso de cubreboca, el distanciamiento social y el evitar salir de los domicilios a exponerse a la convivencia directa con otras personas. ${ }^{2}$ Afortunadamente, el desarrollo de una solución protectora fue rápido, sustancialmente por el desarrollo tecnológico que se había alcanzado en la producción de vacunas en diferentes plataformas. ${ }^{3}$ De esta forma, y prácticamente al año de haberse notificado los primeros casos de esta nueva enfermedad, se autorizó por la administración de medicamentos y alimentos de los Estados Unidos el uso emergente de la vacuna inaugural contra COVID-19, y el 20 de diciembre de 2020 se inició el primer programa nacional de administración a gran escala, en Israel. ${ }^{4}$

En ese país, a nivel nacional y después de la inmunización, en comparación con el pico previo, se observó un descenso de $77 \%$ en los contagios en las personas de 60 años o más, de $68 \%$ en los de 50 a 59 años, de $62 \%$ en los de 40 a 49 y de $52 \%$ en el grupo de 20 a 39 . Estas proporciones fueron del 68, 32, 23 y $22 \%$ en el número de hospitalizaciones en cada uno de los grupos mencionados y de $67,36,16$ y $17 \%$ respectivamente en el conteo de eventos graves. ${ }^{4}$

En esa misma nación, el tres de abril de 2021, 72.1\% de la población de 16 años o más había obtenido las dos porciones del fármaco de Pfizer BNT162b2, una vacuna que codifica el RNA de la proteína en espiga completa del SARS-CoV-2, requerida para la penetración del virus a las células del ser humano. En la vida real y a nivel poblacional, la tasa de vacunación en la población atendida (dos semanas o más de haber recibido la segunda dosis) fue de 2.1 por 100,000 días persona, comparada con una tasa de 91.5 por 100,000 días persona en los no vacunados, dando una efectividad de la vacuna, ajustada por edad, sexo y semana del calendario, de 96.5\% (IC 95\% 96.3-96.8). ${ }^{5}$ Dicha protección contra muerte relacionada a COVID-19, fue de $100 \%$ en los menores de 45 años y de 96.5 a $98 \%$ en los de 45 o más. Una medida ajustada de su capacidad efectiva de 98.4\% (IC 95\% 98.1-98.6) frente a hospitalización por dicha enfermedad, grave o crítica, de 98\% (IC 95\% 97.7-98.3) en cualquier ingreso hospitalario, de $97.7 \%$ (IC 95\% 97.5-97.9) por este contagio sintomático y de 93.8\% (IC 95\% 93.3-94.2) para contagio por SARSCoV-2 asintomático, lo cual habla de la bondad de un programa de inmunización universal. ${ }^{5}$ No obstante, la posibilidad poco mayor del $6 \%$ de una infección asintomática en personas vacunadas refuerza la necesidad del uso de otras medidas de protección adicional a fin de evitar la infección. ${ }^{6}$
Es interesante señalar que la efectividad de la vacuna, siete días posteriores a la segunda inyección, buscando evitar el contagio sintomático, es inferior en hombres (88\%, IC 95\% 71-98), que en mujeres (96\%, IC 95\% 90-100) y es más baja en el conjunto de 40 a 69 años (90\%, IC 95\% 75-98), que en el grupo de 16 a 39 (99\%, IC 95\% 96-100) o en los de 70 o más (98\%, IC 95\% 90-100). Esa medida es mayor en las personas con obesidad (98\%, IC 95\% 91-100), que en aquellos con hipertensión (95\%, IC 95\% 84-100), diabetes (91\%, IC 95\% 68-100), los que padecen una o dos de estas afecciones (95\%, IC 95\% 88-100) o los que tienen tres o más condiciones coexistentes (89\%, IC 95\% 68-98). ${ }^{7}$

En esta campaña nacional de vacunación en Israel, aún después de la parte inicial del inyectable, se observó una eficacia de la vacuna de 54.9\% (IC 95\%, 21.4-73.6) para infección sintomática de COVID-19, evidente aún en dolientes con inmunosupresión $(49.7 \%$, IC 95\%, 9.2-72.1), trastorno cardiovascular (51.3\%, IC 95\%, 14.4-72.3) o cáncer (55.9\%, IC 95\%, 22.7-74.8). ${ }^{8}$

Una estrategia hacia lograr una superior cobertura con una primera parte de la vacuna y posponer el refuerzo de la siguiente dosis fue implementada por algunos países, como el Reino Unido, donde se observó una reducción de la transmisión del germen con esta propuesta. ${ }^{9}$ En ese país, el programa nacional de inoculación incluyó además la aplicación de un fármaco de otra plataforma, la preparación ChAdOx1 nCoV-19, de Oxford-AstraZeneca, la cual utiliza un adenovirus de chimpancé, logrando expresar una secuencia codificada de la proteína espiga del SARS-CoV-2. En Escocia, con una cobertura dada por la medida inicial de cualquiera de las dos vacunas, de $35.1 \%$ de las mujeres y de $25 \%$ de los hombres, se logró una eficacia en la reducción de la hospitalización, tras 21 días de aplicada la vacuna, de $100 \%$ en los menores de 80 años con la ampolleta ChAdOx1 nCoV-19 y de 63 a 93\% con el elixir BNT162b2, de Pfizer-BioNTech. ${ }^{10}$ En el Reino Unido, la efectividad de tal alternativa BNT162b2, a los dos semanas o más de la segunda aplicación fue de $89 \% .^{11}$

Es relevante señalar que, en este grupo de edad, la medida de la primera ampolleta para reducir el contagio fue de $70 \%$ a partir de cuatro semanas de la mitad inicial de la vacuna BNT162b2, y de $60 \%$ en aquellos que recibieron la parte inicial de la ampolleta ChAdOx1 nCoV19. El riesgo de ingreso al hospital disminuyó $43 \%$ con la primera inyección y $37 \%$ con la segunda y el riesgo de morir disminuyó en 51\% a las dos semanas de la inyección previa de la vacuna BNT162b2. ${ }^{11}$ En el Reino Unido, un modelaje ha proyectado que con una cobertura y efectividad de la inoculación de 100\%, se logrará una disminución de muertes de $62 \%$ en los mayores de 80 
años, de $84 \%$ en los de 70 a 79, de 94\% en los de 60 a 69 , de $98 \%$ en los de 50 a 59 y del $100 \%$ en el grupo de 18 a 50 años. La reducción en hospitalizaciones oscilará del 33\% en los de más de 80 años, a $86 \%$ en los de 50 a 59 y $100 \%$ en los menores de 50 . A su vez, la reducción de ingresos a unidades de cuidados intensivos rondaría $3 \%$ en los de más de 80 años a $78 \%$ en los 50 a 59 y $100 \%$ en los menores de $50 .^{12}$

En los EEUU, la vacunación universal se ha basado en dos vacunas de la plataforma mRNA, la BNT-162b2 de Pfizer-BioNTech y la mRNA-1273 de Moderna. La capacidad efectiva de estos fármacos se ha documentado, siendo de 96.2\% (IC 95\% 95.5-96.9) con la vacuna BNT-162b2, siete o más días después de la segunda parte, y de 98.2\% (IC 95\% 97.5-98.6) con la alternativa mRNA-1273. ${ }^{13}$ La efectividad de ambas vacunas supera 95\% independientemente de la edad, raza, sexo o índice de comorbilidad de Charlson. ${ }^{13}$ En la Unión Americana, con el programa de inmunización se ha logrado una disminución de $69 \%$ de los casos de COVID-19, de 59\% en las consultas del servicio de urgencias, de $63 \%$ en los ingresos hospitalarios y de $80 \%$ en las defunciones. ${ }^{14}$ Es importante indicar que, en esa nación, hacia el mes de mayo de 2021, la cobertura de vacunación con una ampolleta era de $48.3 \%$ y con dos dosis de $38.3 \%$, aunque mayor en la ciudadanía blanca de ese país (45.2\%) que en población negra (32\%) o de ascendencia hispana (30.8\%). ${ }^{15}$ Aún en el profesional de la salud, mientras que sólo $45.6 \%$ de los auxiliares habían recibido un esquema completo de inoculación, $58.5 \%$ del personal de dietología, lavandería o mantenimiento lo había recibido, 56.7\% de las enfermeras, $69.2 \%$ de los terapistas y $75.1 \%$ de los médicos. ${ }^{16}$

En Chile, la campaña de vacunación se realizó con un fármaco de virus completos inactivados, la marca CoronaVac. Con esta alternativa se logró una cobertura con dos inyecciones de 41\%, más un 5.3\% adicional que recibió sólo una aplicación. La cobertura con dos ampolletas fue superior en mujeres (44.3\%) que en hombres (37.1\%) y arriba de $80 \%$ en las personas de 60 años o más, así como en los que tenían una o más condiciones de salud asociadas (64.6 versus $29.6 \%) .{ }^{17}$ La efectividad de la ampolleta con dos partes frente a la infección por SARS-CoV-2 fue de 65.9\% (IC 95\% 65.2-66.6), para hospitalización de 87.5\% (IC 95\% 86.7-88.2), para ingreso a la Unidad de Cuidados Intensivos de $90.3 \%$ (IC 95\% 89.1-91.4) y contra muerte de $86.3 \%$ (IC 95\% 84.5-87.8). ${ }^{17}$

\section{Trabajadores de la salud}

En EEUU, en población trabajadora del área de la salud y de primera línea, la efectividad de una inyección de la vacuna BNT162b2 fue de $80 \%$ (IC 95\% 60-90) y con las dos partes de 93\% (IC 95\% 78-98), mientras que con la opción mRNA-1273 estos valores fueron de 83\% (IC $95 \%$ 40-95) y $82 \%$ (IC 95\% 20-96). ${ }^{18}$ En la Universidad de California, en 10,188 trabajadores se observó una reducción en el número de casos de COVID-19 de 55\% luego de una semana de la aplicación de la segunda inyección de la variedad BNT162b2, de 84\% después de dos semanas y de $94 \%$ tras tres semanas de la segunda dosis de la vacuna. ${ }^{19}$

En el Reino Unido, la efectividad de la vacuna BNT162b2 fue de 70\% luego de 14 días de la administración inicial, ${ }^{20}$ mientras que en Brasil, en trabajadores de la salud, esa medida en la marca CoronaVac fue de 49.4\% (IC 95\% 13.2-71.9) en las dos semanas tras recibir la primera punción del fármaco, y de 35\% (IC 95\% 6.6-60.5) tras dos semanas de la dosificación original y de $37.9 \%$ (IC 95\% 46.4-73.6) dos semanas después de la segunda ampolleta. ${ }^{21}$

\section{La vacuna ante las variantes del virus SARS-CoV-2}

En julio de 2021, en una comunidad del estado de Massachusetts en los Estados Unidos, se identificaron 469 casos de COVID-19 asociados con eventos y reuniones de un número importante de personas, con una cobertura de vacunación de 69\%. De esos sucesos, $74 \%$ ocurrieron en sujetos que tenían dos dosis de una tecnología mRNA o una sola de la oferta de Janssen (Johnson \& Johnson), más de dos semanas antes de la exposición. En 89\% de las incidencias se identificó la variante B.1.617.2 (Delta) del germen. De los individuos vacunados, $79 \%$ fueron sintomáticos, pero no hubo defunción alguna. ${ }^{22}$

En Inglaterra, en una casa de cuidados de adultos longevos se observó una tasa de ataque de 35.7\% en el personal y de $81.3 \%$ en los residentes, que habían

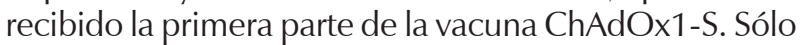
$10 \%$ de los casos fue hospitalizado y ninguno falleció. ${ }^{23}$

En Francia, con dos porciones de un inyectable mRNA se advirtió una efectividad de 88\% (IC 95\% 81-92) contra el patógeno SARS-CoV-2 original, de $86 \%$ (IC 95\% 81-90) frente a la variante B.1.1.7 y de 77\% (IC $95 \%$ 63-86) frente a la variante B.1.351/P.1. ${ }^{24}$ Los sujetos que recibieron dos punciones de la alternativa BNT162 b2 mostraron una buena respuesta humoral ante las variantes del virus, aunque con niveles reducidos de anticuerpos neutralizantes en contraste con la variante de Sudáfrica. ${ }^{25}$ No obstante, en Inglaterra se observó que, a pesar de una reducción de cinco veces en los títulos de dichos anticuerpos contra la variante B.1.351, de 
los sujetos vacunados seronegativos 92\% tuvieron títulos mayores de 20 para dicha variante, lo que implica algo de protección. ${ }^{26}$ De hecho, se ha observado respuesta adecuada de los mencionados anticuerpos frente a las nuevas variantes B.1.429, B.1.526 y B.1.1.7. ${ }^{27}$

En Inglaterra, la efectividad de dos ampolletas de la vacuna BNT162b2 fue menor, enfrentada a la variante Delta (88\% IC 95\% 85.3-90.1), que contra la variante Alfa (93.7\% IC 95\% 91.6-95.3). Con dos dosis de la opción ChAdOx1 nCoV-19, la medición también fue menor opuesta al contagio de la Delta $(67 \%$ IC 95\% 61.3-71.8) que contra la Alfa (74.5\% IC 95\% 68.479.4). ${ }^{28}$ En Escocia, a su vez, la efectividad de la opción BNT162b2 frente a la Delta fue de 79\% (IC 95\% 75-82), mientras que con la competidora ChAdOx1 nCoV-19 la cifra fue de $60 \%$ (IC 95\% 53-66). ${ }^{29}$

\section{Vacunación en poblaciones especiales}

Quienes sufren de padecimiento terminal de riñón tienen el doble de riesgo de fallecer ante un contagio por SARS-CoV-2. ${ }^{1}$ La inoculación con dos ampolletas de la vacuna mRNA-BNT162b2 ha mostrado una buena seroconversión en cerca de $98 \%$ de los individuos vacunados, con adecuada seguridad y tolerancia. ${ }^{30} \mathrm{Si}$ bien la seroconversión en los enfermos en diálisis es superior a 95\%, en los pacientes con trasplante renal se ve muy afectada (42\%). Los enfermos con trastorno renal vacunados tienen mejor réplica de seroconversión con la alternativa mRNA-1273 que con la BNT162b2, tanto en pacientes en diálisis (97 versus $88 \%$ ) como en aquellos con riñón trasplantado (49 versus $26 \%$ ). ${ }^{31}$ La pobre respuesta a la vacuna BNT162b2 también se ha visto en enfermos con trasplante hepático, con sólo $47.5 \%$ de los vacunados quienes desarrollan anticuerpos neutralizantes contra el virus. ${ }^{32}$ Una tercera dosis de la mezcla mRNA-1273 pudiera inducir una respuesta superior en afectados con trasplante renal ${ }^{33} \mathrm{O}$ con otros trasplantes de órganos. ${ }^{34}$

En pacientes con cáncer, dos inyecciones de la opción BNT162b2 logran una adecuada respuesta inmunológica, de forma segura y sin toxicidad. ${ }^{35}$ A su vez, en dolientes con enfermedad reumática autoinmune, la marca CoronaVac ha revelado ser inmunogénica y segura. ${ }^{36}$

En la mujer embarazada, la vacunación con tecnología de RNA mensajero ha mostrado ser segura y eficaz. ${ }^{37}$

\section{CONCLUSIONES}

Ha habido un rápido progreso de vacunas que protejan contra la infección por SARS-CoV-2, en gran medida basado en el avance tecnológico alcanzado antes de la pandemia en la disponibilidad de diferentes plataformas dedicadas al desarrollo de vacunas. Los ensayos clínicos oportunos y bien conducidos permitieron probar vacunas que han alcanzado buena inmunogenicidad, eficacia, eficiencia y seguridad. Los estudios a nivel poblacional han mostrado el impacto de la inmunización universal. No obstante, es indispensable continuar con medidas de protección adicionales, buscando evitar la exposición al virus y con ello favorecer su circulación en población susceptible. La inmunidad adquirida por la afección, sumada a la conseguida con la vacunación universal y las medidas de contención poblacionales adicionales (verbigracia, uso de cubreboca, sana distancia, evitar reuniones y concentraciones masivas), ayudarán a dejar atrás la carga tan significativa que ha provocado en la salud pública global esta pandemia que se enfrenta. Queda claro que ninguna medida por sí sola es suficiente para impedir la circulación del patógeno y la propagación de la enfermedad.

\section{REFERENCIAS}

1. Escobedo-de la Peña J, Rascón Pacheco RA, Ascencio-Montiel IJ, González-Figueroa E, Fernández-Gárate JE, Medina-Gómez OS et al. Hypertension, diabetes and obesity, major risk factors for death in patients with COVID-19 in Mexico. Arch Med Res. 2021; 52 (4): 443-449. Available in: https://doi.org/10.1016/j. arcmed.2020.12.002

2. Rabold $\mathrm{E}$, Rovnan $\mathrm{H}$, DuMont $\mathrm{T}$. Isolation requirements and personal protective equipment in the COVID-19 pandemic. Crit Care Nurs Q. 2020; 43 (4): 407-412. Available in: https://doi. org/10.1097/CNQ.0000000000000326

3. Excler JL, Saville M, Berkley S, Kim JH. Vaccine development for emerging infectious diseases. Nat Med. 2021; 27 (4): 591-600. Available in: https://doi.org/10.1038/s41591-021-01301-0

4. Rossman H, Shilo S, Meir T, Gorfine M, Shalit U, Segal E. COVID-19 dynamics after a national immunization program in Israel. Nat Med. 2021; 27: 1055-1061. Available in: https://doi. org/10.1038/s41591-021-01337-2

5. Haas EJ, Angulo FJ, McLaughlin JM, Anis E, Singer SR, Khan F et al. Impact and effectiveness of mRNA BNT162b2 vaccine against SARS-CoV-2 infections and COVID-19 cases, hospitalisations, and deaths following a nationwide vaccination campaign in Israel: an observational study using national surveillance data. Lancet. 2021; 397: 1819-1829. Available in: https://doi.org/10.1016/S01406736(21)00947-8

6. Alagoz O, Sethi AK, Patterson BW, Churpek M, Alhanaee G, Scaria E, Safdar N. The impact of vaccination to control COVID-19 burden in the United States: a simulation modeling approach. medRxiv. 2021.03.22.21254131. Available in: https://doi. org/10.1101/2021.03.22.21254131

7. Dagan N, Barda N, Kepten E, Miron O, Perchik S, Katz MA et al. BNT162b2 mRNA Covid-19 vaccine in a nationwide mass vaccination setting. N Engl J Med. 2021; 384: 1412-1423. Available in: https://doi.org/10.1056/NEJMoa2101765

8. Chodick G, Tene L, Patalon T, Gazit S, Tov AB, Cohen D, Muhsen $\mathrm{K}$. Assessment of effectiveness of 1 dose of BNT162b2 vaccine for SARS-CoV-2 infection 13 to 24 days after immunization. JAMA 
Network Open. 2021; 4 (6): e2115985. Available in: https://doi. org/10.1001/jamanetworkopen.2021.15985

9. Jones NK, Rivett L, Seaman S, Samworth RJ, Warne B, Workman $C$ et al. Single-dose BNT162b2 vaccine protects against asymptomatic SARS-CoV-2 infection. eLife. 2021; 10: e68808. Available in: https://doi.org/10.7554/eLife.68808

10. Vasileiou E, Simpson CR, Shi T, Kerr S, Agrawal U, Akbari A et al. Interim findings from first-dose mass COVID-19 vaccination roll-out and COVID-19 hospital admissions in Scotland: a national prospective cohort study. Lancet. 2021; 397: 1646-1657. Available in: https://doi.org/10.1016/S0140-6736(21)00677-2

11. Lopez BJ, Andrews N, Gower C, Robertson C, Stowe J, Tessier $E$ et al. Effectiveness of the Pfizer-BioNTech and OxfordAstraZeneca vaccines on COVID-19 related symptoms, hospital admissions, and mortality in older adults in England: test negative case-control study. BMJ. 2021; 373: n1088. Available in: http:// dx.doi.org/10.1136/bmj.n1088

12. Cook TM, Roberts JV. Impact of vaccination by priority group on UK deaths, hospital admissions and intensive care admissions from COVID-19. Anaesthesia. 2021; 76: 608-616. Available in: http://dx.doi.org/10.1111/anae.15442

13. Butt AA, Omer SB, Yan P, Shaikh OS, Mayr FB. SARS-CoV-2 vaccine effectiveness in a high-risk national population in a realworld setting. Ann Intern Med [Internet]. 2021; [Epub ahead of print 20 July 2021]. Available in: http://dx.doi.org/doi:10.7326/M21-1577

14. Christie A, Henley SJ, Mattocks L, Fernando R, Lansky A, Ahmad FB et al. Decreases in COVID-19 cases, emergency department visits, hospital admissions, and deaths among older adults following the introduction of COVID-19 vaccine-United States, September 6, 2020-May 1, 2021. MMWR Morb Mortal Wkly Rep. 2021; 70: 858-864. Available in: http://dx.doi.org/10.15585/mmwr. $\mathrm{mm} 7023 \mathrm{e} 2$

15. Pingali $\mathrm{C}$, Meghani $\mathrm{M}$, Razzaghi $\mathrm{H}$, Lamias MJ, Weintraub $\mathrm{E}$, Kenigsberg TA et al. COVID-19 vaccination coverage among insured persons aged $\geq 16$ years, by race/ethnicity and other selected characteristics-eight integrated health care organizations, United States, December 14, 2020-May 15, 2021. MMWR Morb Mortal Wkly Rep. 2021; 70: 985-990. Available in: http://dx.doi. org/10.15585/mmwr.mm7028a1

16. Lee JT, Althomsons SP, Wu H, Budnitz DS, Kalayil EJ, Lindley MC et al. Disparities in COVID-19 vaccination coverage among health care personnel working in long-term care facilities, by job category, National Healthcare Safety Network-United States, March 2021. MMWR Morb Mortal Wkly Rep. 2021; 70: 1036-1039. Available in: http://dx.doi.org/10.15585/mmwr.mm7030a2

17. Jara A, Undurraga EA, González C, Paredes F, Fontecilla T, Jara $G$ et al. Effectiveness of an inactivated SARS-CoV-2 vaccine in Chile. New Engl J Med [Internet]. 2021; [Epub ahead of print 7 July 2021]. Available in: http://dx.doi.org/10.1056/NEJMoa2107715

18. Thompson MG, Burgess JL, Naleway AL, Tyner H, Yoon SK, Meece $\mathrm{J}$ et al. Prevention and attenuation of COVID-19 with the BNT162b2 and mRNA-1273 vaccines. N Engl J Med. 2021; 385: 320-329. Available in: http://dx.doi.org/10.1056/NEJMoa2107058

19. Gohil SK, Olenslager K, Quan KA, Dastur CK, Afsar N, Chang W et al. Asymptomatic and symptomatic COVID-19 infections among health care personnel before and after vaccination. JAMA Network Open. 2021; 4 (7): e2115980. Available in: https://doi.org/10.1001/ jamanetworkopen.2021.15980

20. Azamgarhi T, Hodgkinson M, Shah A, Skinner JA, Hauptmannova I, Briggs TWR et al. BNT162b2 vaccine uptake and effectiveness in UK healthcare workers- a single centre cohort study. Nat Commun. 2021; 12: 3698. Available in: https://doi.org/10.1038/s41467-02123927-x

21. Hitchings MDT, Ranzani OT, Scaramuzzini TMS, Barbosa OS, Almiron $\mathrm{M}$ et al. Effectiveness of CoronaVac among healthcare workers in the setting of high SARS-CoV-2 gamma variant transmission in Manaus, Brazil: a test-negative case-control study. Lancet Reg Health-Americas. 2021:100025. Available in: https:// doi.org/10.1016/j.lana.2021.100025

22. Brown CM, Vostok J, Johnson H, Burns M, Gharpure R, Sami S et al. Outbreak of SARS-CoV-2 infections, including COVID-19 vaccine breakthrough infections, associated with large public gatherings-Barnstable County, Massachusetts, July 2021. MMWR Morb Mortal Wkly Rep. 2021; 70 (31): 1059-1062. Available in: http://dx.doi.org/10.15585/mmwr.mm7031e2

23. Williams SV, Vusirikala A, Ladhani SN, Fernandez R, De Olano $\mathrm{E}$, lyanger $\mathrm{N}$ et al. . An outbreak caused by the SARS-CoV-2 Delta (B.1.617.2) variant in a care home after partial vaccination with a single dose of the COVID-19 vaccine Vaxzevria, London, England, April 2021. Euro Surveill. 2021; 26 (27): pii=2100626. Available in: https://doi.org/10.2807/1560-7917. ES.2021.26.27.2100626

24. Charmet T, Schaeffer L, Grant R, Galmiche S, Chény O, Von Platen C et al. Impact of original, B.1.1.7, and B.1.351/P.1 SARSCoV-2 lineages on vaccine effectiveness of two doses of COVID-19 mRNA vaccines: Results from a nationwide case-control study in France. Lancet Reg Health-Europe. 2021; 100171. Available in: https://doi.org/10.1016/j.lanepe.2021.100171

25. Becker M, Dulovic A, Junker D, Ruetalo N, Kaiser PD, Pinilla YT et al. Immune response to SARS-CoV-2 variants of concern in vaccinated individuals. Nat Commun. 2021; 12: 3109. Available in: https://doi.org/10.1038/s41467-021-23473-6

26. Jalkanen $P$, Kolehmainen $P$, Hakkinen HK, Huttunen M, Tahtinen PA, Lundberg $R$ et al. COVID-19 mRNA vaccine induced antibody responses against three SARS-CoV-2 variants. Nat Commun. 2021; 12: 3991. Available in: https://doi.org/10.1038/s41467-02124285-4

27. Liu Y, Liu J, Xia H, Zhang X, Zou J, Fontes-Garfias CR et al. BNT162b2-elicited neutralization against new SARS-CoV-2 spike variants. N Engl J Med. 2021; 385: 472-474. Available in: https:// doi.org/10.1056/NEJMc2106083

28. Lopez BJ, Andrews N, Gower C, Gallagher E, Simmons R, Thelwall $S$ et al. Effectiveness of Covid-19 vaccines against the B.1.617.2 (Delta) variant. New Engl J Med [Internet]. 2021; [Epub ahead of print 21 July 2021]. Available in: https://doi.org/10.1056/ NEJMoa2108891

29. Sheikh A, McMenamin J, Taylor B, Robertson C, on behalf of Public Health Scotland and the EAVE II Collaborators. SARS-CoV-2 Delta VOC in Scotland: demographics, risk of hospital admission, and vaccine effectiveness. Lancet. 2021; 397: 2461-2462. Available in: https://doi.org/10.1016/S0140-6736(21)01358-1

30. Zitt E, Davidovic T, Schimpf J, Abbassi-Nik A, Mutschlechner $\mathrm{B}$, Ulmer $\mathrm{H}$ et al. The safety and immunogenicity of the mRNABNT162b2 SARS-CoV-2 vaccine in hemodialysis patients. Front Immunol. 2021; 12: 704773. Available in: https://doi.org/10.3389/ fimmu.2021.704773

31. Stumpf J, Siepmann T, Lindner T, Karger C, Schwobel J, Anders L et al. Humoral and cellular immunity to SARS-CoV-2 vaccination in renal transplant versus dialysis patients: a prospective, multicenter observational study using mRNA-1273 or BNT162b2 mRNA vaccine. Lancet Reg Health - Europe 2021; [Epub ahead of print 23 July 2021]: 100178. Available in: https://doi.org/10.1016/j. lanepe.2021.100178

32. Rabinowich L, Grupper A, Baruch R, Ben-Yehoyada M, Halperin $\mathrm{T}$, Turner $\mathrm{D}$ et al. Low immunogenicity to SARS-CoV-2 vaccination among liver transplant recipients. J Hepatol. 2021; 75: 435-438. Available in: https://doi.org/10.1016/j.jhep.2021.04.020

33. Benotmane I, Gautier G, Perrin P, Olagne J, Cognard N, FafiKremer $S$ et al. Antibody response after a third dose of the mRNA1273 SARS-CoV-2 vaccine in kidney transplant recipients with 
Plast Restaur Neurol. 2021;8 (1): 13-18

minimal serologic response to 2 doses. JAMA. 2021; Published online July 23, 2021. Available in: https://doi.org/10.1001/ jama.2021.12339

34. Kamar N, Abravanel F, Marion O, Couat C, Izopet J, Del Bello A. Three doses of an mRNA Covid-19 vaccine in solid-organ transplant recipients. N Engl J Med [Internet]. 2021; [Epub ahead of print 23 June 2021]. Available in: https://doi.org/10.1056/NEJMc2108861

35. Monin L, Laing AG, Muñoz-Ruiz M, McKenzie DR, del Molino del Barrio I, Alaguthurai T et al. Safety and immunogenicity of one versus two doses of the COVID-19 vaccine BNT162b2 for patients with cancer: interim analysis of a prospective observational study. Lancet Oncol. 2021; 22: 765-778. Available in: https://doi. org/10.1016/S1470-2045(21)00213-8
36. Medeiros-Ribeiro AC, Aikawa NE, Saad CGS. et al. Immunogenicity and safety of the CoronaVac inactivated vaccine in patients with autoimmune rheumatic diseases: a phase 4 trial. Nat Med [Internert] 2021; [Epub ahead of print 30 Jul 2021]. Available in: https://doi.org/10.1038/s41591-021-01469-5

37. Shimabukuro TT, Kim SY, Myers TR, Moro PL, Oduyebo T, Panagiotakopoulos $L$ et al. Preliminary findings of mRNA Covid-19 vaccine safety in pregnant persons. N Engl J Med. 2021; 384: 2273-2282. Available in: https://doi.org/10.1056/NEJMoa2104983

Conflicto de intereses: No existe conflicto de interés ni apoyos extraordinarios. 